\title{
Radiation Reaction: General approach and applications, especially to electrodynamics
}

\author{
R. F. O'Connell \\ Department of Physics and Astronomy, \\ Louisiana State University, Baton Rouge, LA 70803-4001 USA
}

(Dated: August 22, 2018)

\begin{abstract}
Radiation reaction (but, more generally, fluctuations and dissipation) occurs when a system interacts with a heat bath, a particular case being the interaction of an electron with the radiation field. We have developed a general theory for the case of a quantum particle in a general potential (but, in more detail, an oscillator potential) coupled to an arbitrary heat bath at arbitrary temperature, and in an external time-dependent $c$-number field. The results may be applied to a large variety of problems in physics but we concentrate by showing in detail the application to the blackbody radiation heat bath, giving an exact result for radiation reaction problem which has no unsatisfactory features such as the runaway solutions associated with the Abraham-Lorentz theory. In addition, we show how atomic energy and free energy shifts due to temperature may be calculated. Finally, we give a brief review of applications to Josephson junctions, quantum statistical mechanics, mesoscopic physics, quantum information, noise in gravitational wave detectors, Unruh radiation and the violation of the quantum regression theorem.
\end{abstract}




\section{INTRODUCTION}

Radiation reaction is familiar to most readers through the Abraham-Lorentz equation for a radiating electron [1-3]. It arises from the fact that electric and magnetic fields emitted by an accelerating electron act back on the electron, resulting in a retarding force. However, the equation obtained by Abraham [1] and Lorentz [2] displays unphysical runaway solutions. Through the years, there have been many attempts to obtain a more satisfactory result but none were without difficulties of one kind or another until in 1991 we proposed a physically consistent solution in the form of a quantum Langevin equation [4]. We discuss that result below in Sec. III, but first we consider more general properties of dissipation and fluctuations, since they appear in many areas of physics.

The equation of motion of a quantum particle in a heat bath (also referred to as a reservoir or as the environment) was pioneered in 1965 by Ford, Kac and Mazur [5]. These authors used a quantum model of coupled oscillators to obtain a quantum Langevin equation that showed explicitly the role of fluctuation and radiation reaction (dissipation) effects. By 1983, there was much general interest in mesoscopic systems and Caldeira and Leggett [6] showed that quantum heat baths could play an important role in the analysis of such systems by analyzing in detail the effect of dissipation on quantum tunneling in such systems as a single Josephson junction or a SQUID. These authors, again considering an Ohmic heat bath, used the path integral influence functional approach of Feynman and Vernon [7], a technique which was then followed by many investigators, particularly these working on coherence and entanglement problems. By contrast, the present authors, in collaboration with J. T. Lewis, developed a general quantum Langevin equation approach to these problems [8, 9] that, in our opinion, is simpler and more physically transparent. In particular, we went beyond the Ohmic model to consider an arbitrary frequency-dependent heat bath (in particular a blackbody radiation field heat bath).

In general, coupling a system to a heat bath produces two related effects: dissipation and fluctuations. The study of these phenomena is loosely referred to as the "Brownian motion," problem, going back to the work of Robert Brown, a Scottish botanist who in 1828 and 1829 published his work on the random motion of pollen grains immersed in a fluid [10]. The explanation of Brown's phenomenon was given by Einstein and Smoluchowski in the first decade of the twentieth century $[11,12]$. This work helped to definitively establish 
the atomic theory of matter since the irregular motion was clearly identified as being due to collisions with the molecules in the liquid. The term "Brownian motion," is now used in a generic sense to denote random motion and it covers a wide spectrum of phenomena from the motion of very fine particles suspended in a gas to the motion of electrons in a blackbody radiation heat bath.

Einstein's explanation of Brownian motion used a discrete time approach. In particular, his results included an explanation of the fact that increasing temperatures lead to more agitated Brownian motion, in a relation in which the diffusion coefficient is shown to be equal to the product of $k T$ and the mobility, perhaps the first example of what is now called the fluctuation-dissipation theorem.

Shortly after the work of Einstein and Smoluchowski, Langevin [13] presented a different approach to the subject which, in the words of Chandrasekhar [14], constitutes the " modern," approach to this and other such problems. The essence of Langevin's approach is a continuous time approach implemented by adoption of a stochastic differential equation, i.e., an equation for quantities which are random in nature. In other words, Langevin provided an elegant solution to the problem of generalizing a dynamical equation to a probabilistic equation. This was to be the start of a major new field of study with applications in physics, chemistry, biology, and many other fields.

The approach of Langevin was phenomenological but its essential correctness has been verified by various microscopic studies. A key feature of his approach was to separate the total force acting on a particle due to its environment into two parts: a mean frictional force and a fluctuation (random) force. These forces are related to each other as a consequence of the requirement that the equilibrium state be stationary. On the other hand these two forces are very different in nature: The fluctuation force is basically microscopic in nature and has a time scale determined by the mean time between collisions, whereas the time scale of the frictional force is proportional to the self-diffusion constant and is much larger. Explicitly, Langevin explained the motion of a "Brownian," particle (an otherwise free particle in a dissipative environment) by an elegant stochastic classical differential equation [13]:

$$
m \ddot{x}+\zeta \dot{x}=F(t)
$$

where $m$ and $x$ denote the mass and coordinate of the particle, respectively, and the dot denotes differentiation with respect to time. The force on the particle consists of the frictional 
(dissipative) term $-\zeta \dot{x}$ and the random (fluctuation or noise) term $F(t)$. The latter term is zero at zero temperature, in contrast to the corresponding situation in quantum mechanics, as we shall see later.

Since the past motion does not appear in the Langevin equation (1), one says that there is no memory or, equivalently (in the classical case under discussion but not in the quantum case to be discussed later), we say that the process is Markovian. In addition, the autocorrelation of the random force is a $\delta$ function (a manifestation of the Markovian process) and is also proportional to $\zeta$. The latter result is a manifestation of the fluctuationdissipation theorem.

In addition, with the usual definition of the diffusion constant,

$$
D \equiv \frac{1}{2} \lim _{t \rightarrow \infty} \dot{s}(t)
$$

where

$$
s\left(t-t^{\prime}\right)=\left\langle\left[x(t)-x\left(t^{\prime}\right)\right]^{2}\right\rangle
$$

is the mean-square displacement and the angular brackets denote the average with respect to the canonical ensemble of the system, Langevin readily obtained the famous Einstein relation

$$
D=\frac{k T}{\zeta}
$$

As noted above, this is an example of an intimate connection between fluctuations and dissipation. Another example was provided by Nyquist who showed that the random fluctuations in voltage across a resistor measured by Johnson are determined by its impedance (the famous Johnson-Nyquist noise [15] in electrical circuits). A general quantum formulation of the fluctuation-dissipation theorem first appeared in the well known article of Callen and Welton [16].

We turn now to another key property of the Langevin equation: Whereas the original Brownian motion experiment and the analysis of it pertained to a system in thermal equilibrium, the Langevin equation can be generalized in a simple manner to include an external force on the right hand side and thus can describe the irreversible approach to equilibrium.

The Langevin equation is phenomenological. As noted above, its derivation from a quantum microscopic theory was first given by Ford, Kac and Mazur [5]. Then in 1986, in an article entitled "Quantum Langevin Equation", [9] together with J. T. Lewis we gave a detailed discussion and presented the general form of the equation consistent with fundamental 
physical requirements, in particular causality and the second law of thermodynamics. Based on these conclusions, we were able to obtain the most general quantum Langevin equation [given below in (6)] for the macroscopic description of a quantum particle with passive dissipation and moving in an arbitrary external potential. We then showed that the most general form can be realized by a simple oscillator model of a heat bath. For the purpose of this article, it is convenient to use this model, the so-called independent-oscillator (IO) model, for which the quantum Hamiltonian is

$$
H_{\mathrm{IO}}=\frac{p^{2}}{2 m}+V(x)+\sum_{j}\left(\frac{p_{j}^{2}}{2 m_{j}}+\frac{1}{2} m_{j} \omega_{j}^{2}\left(q_{j}-x\right)^{2}\right)-x f(t) .
$$

Here, $V(x)$ is a particle potential energy, $f(t)$ is an external applied force, while $x$ and $p$ are the particle position and momentum operators and $q_{j}$ and $p_{j}$ are those for the $j$ 'th bath oscillator. The parameters are $m$, the particle mass, and $m_{j}$ and $\omega_{j}$, the mass and frequency of the $j$ 'th bath oscillator.

The procedure used in obtaining the corresponding Langevin equation from the microscopic Hamiltonian is common to all such problems and consists of use of the Heisenberg equations of motion to obtain the equations of motion for both the dynamical variables of the particle $(x, p)$ and the dynamical variables of the heat bath $\left(q_{j}, p_{j}\right)[9]$. These are coupled equations and the next step is to eliminate the bath variables. This leads to an inhomogeneous differential equation for the $q_{j}$. Then, typical of the way that the timereversal invariance of the original equations is broken in macroscopic equations, one chooses the retarded solution of this equation. This solution for $q_{j}$ is then substituted into the equation for $x$ to get the Langevin equation for a quantum particle of mass $m$ moving in a one dimensional potential $V(x)$ in an arbitrary heat bath and temperature $T$ :

$$
m \ddot{x}+\int_{-\infty}^{t} d t_{1} \mu\left(t-t_{1}\right) \dot{x}\left(t_{1}\right)+V^{\prime}(x)=F(t)+f(t),
$$

where the dot and prime denote, respectively, the derivative with respect to $t$ and $x$. This is a Heisenberg equation of motion for the coordinate operator $x$. The coupling with the heat bath is described by two terms: an operator-valued random force $F(t)$ with mean zero, and a mean force characterized by the memory function $\mu(t)$. These quantities are given in terms of the heat bath variables:

$$
\mu(t)=\sum_{j} m_{j} \omega_{j}^{2} \cos \left(\omega_{j} t\right) \Theta(t)
$$


where $\Theta(t)$ is the Heaviside step function (by convention the memory function vanishes for negative times), and

$$
F(t)=\sum_{j} m_{j} \omega_{j}^{2} q_{j}^{h}(t)
$$

where $q_{j}^{h}(t)$ denotes the general solution of the homogeneous equation for the heat bath oscillators (corresponding to no interaction). Using these results, we find that the (symmetric) autocorrelation of $F(t)$ is

$$
\frac{1}{2}\left\langle F(t) F\left(t^{\prime}\right)+F\left(t^{\prime}\right) F(t)\right\rangle=\frac{1}{\pi} \int_{0}^{\infty} d \omega \operatorname{Re}\left[\tilde{\mu}\left(\omega+i 0^{+}\right)\right] \hbar \omega \operatorname{coth} \frac{\hbar \omega}{2 k T} \cos \left[\omega\left(t-t^{\prime}\right)\right]
$$

and the nonequal-time commutator of $F(t)$ is

$$
\left[F(t), F\left(t^{\prime}\right)\right]=\frac{2}{i \pi} \int_{0}^{\infty} d \omega \operatorname{Re}\left[\tilde{\mu}\left(\omega+i 0^{+}\right)\right] \hbar \omega \sin \left[\omega\left(t-t^{\prime}\right)\right] .
$$

In these expressions

$$
\tilde{\mu}(z)=\int_{0}^{\infty} d t e^{i z t} \mu(t), \quad \operatorname{Im} z>0
$$

is the Fourier transform of the memory function $\mu(t)$. Finally, $F(t)$ has the Gaussian property: correlations of an odd number of factors of $F$ vanish; those of an even number of factors are equal to the sum of products of pair correlations, the sum being over all pairings with the order of the factors preserved within each pair. Equation (9) is an exact fluctuationdissipation theorem and we emphasize that it is independent of both the potential $V(x)$ and the external force $f(t)$. Physically, it expresses the fact that the spontaneous equilibrium fluctuations of the heat bath (described by the left side of (9)) are related to the dissipation parameter $\operatorname{Re} \tilde{\mu}(\omega)$.

From the explicit expression (7) it is clear that the memory function is dependent only on the bath parameters. We obtained a key constraint on this function by considering the effect of an arbitrary $c$-number external force $f(t)$ acting on an otherwise free particle $(V(x)=0)$ [9]. If we assume that $f(t)$ vanishes in the distant past and future, the effect is to carry the system of free particle coupled to the bath in a complete cycle from a state of equilibrium, through a continuous sequence of intermediate states, and back to equilibrium. The second law of thermodynamics, in the Kelvin-Planck form, then requires that the net work done by this force be positive, which in turn requires that the spectral distribution $\operatorname{Re}\left[\tilde{\mu}\left(\omega+i 0^{+}\right)\right]$ must satisfy the positivity condition:

$$
\operatorname{Re}\left\{\tilde{\mu}\left(\omega+i 0^{+}\right)\right\} \geq 0, \quad-\infty<\omega<\infty
$$


We note that since the memory function is real the spectral distribution must also satisfy the reality condition: $\operatorname{Re}\left[\tilde{\mu}\left(-\omega+i 0^{+}\right)\right]=\operatorname{Re}\left[\tilde{\mu}\left(\omega+i 0^{+}\right)\right]$.

The positivity condition (12) together with the fact, obvious from the definition (11), that $\tilde{\mu}(z)$ is analytic in the upper half plane, means that means that $\tilde{\mu}(z)$ must be what is called a positive real function. This is a very restricted class of functions of a complex variable, with special properties which include the Stieltjes inversion theorem:

$$
\tilde{\mu}(z)=\frac{2 i z}{\pi} \int_{0}^{\infty} d \omega \frac{\operatorname{Re}\left\{\tilde{\mu}\left(\omega+i 0^{+}\right)\right\}}{z^{2}-\omega^{2}} .
$$

Thus, we see that in the quantum Langevin equation the memory function as well as the correlation and commutator of the random force, are completely characterized by the spectral distribution.

There are further conditions on the spectral function. As a consequence of the inversion theorem (13) we see that

$$
\int_{0}^{\infty} d \omega \frac{\operatorname{Re}\left\{\tilde{\mu}\left(\omega+i 0^{+}\right)\right\}}{1+\omega^{2}}<\infty .
$$

An important further constraint is what has been called the zero'th law of thermodynamics: there must be an equilibrium state. This requires that the spectral distribution must be everywhere positive, with no gaps in which it vanishes. This in turn requires for our model that the bath frequencies must be infinite in number and continuously distributed.

In the case of an oscillator potential $V(x)=\frac{1}{2} K x^{2}$, the solution of the quantum Langevin equation (6) is given by [8]

$$
\tilde{x}(\omega)=\alpha(\omega)[\tilde{F}(\omega)+\tilde{f}(\omega)],
$$

where the superposed tilde to denotes the Fourier transform, e.g., $\tilde{x}(\omega)$ is the Fourier transform of the operator $x(t)$, and $\alpha(\omega)$ is the generalized susceptibility (a $c$-number) given by

$$
\alpha(\omega)=\frac{1}{-m \omega^{2}-i \omega \tilde{\mu}(\omega)+K} .
$$

As a simple application of this result, we find for the autocorrelation and commutator of the operator $x(t)$, in linear response, are $[8,17]$

$$
\begin{aligned}
\frac{1}{2}\left\langle x(t) x\left(t^{\prime}\right)+x\left(t^{\prime}\right) x(t)\right\rangle & =\frac{\hbar}{\pi} \int_{0}^{\infty} d \omega \operatorname{Im}\left\{\alpha\left(\omega+i 0^{+}\right)\right\} \operatorname{coth} \frac{\hbar \omega}{2 k T} \cos \left(\omega\left(t-t^{\prime}\right)\right), \\
{\left[x(t), x\left(t^{\prime}\right)\right] } & =\frac{2 \hbar}{i \pi} \int_{0}^{\infty} d \omega \operatorname{Im}\left\{\alpha\left(\omega+i 0^{+}\right)\right\} \sin \omega\left(t-t^{\prime}\right) .
\end{aligned}
$$


Equations (9), (10) and (17) are exact fluctuation-dissipation theorems for our general analysis and they provide the foundation for subsequent developments. We note, however, that equation (17) depends on both the potential and the dissipative parameter. In fact, in the classical case (but not in the quantum case), we showed that, in the case of a weak applied force, the spontaneous fluctuations (described by the left-side of (17)) relax with the same time constant as the induced (by $f(t)$ ) non-equilibrium fluctuations [18]. This is the essence of the Onsager regression hypothesis [19] which states that regression of fluctuations is governed by macroscopic equations describing the approach to equilibrium.

We have now essentially all the tools that we need and thus it is time to turn to specific applications. However, here we decided to concentrate on important problems in electrodynamics. First, in section II, we survey the vast amount of work on the problem of runaway solutions of the Abraham-Lorentz equation for a radiating electron. Many of these attempts started with force equations which do not encapsulate the important time development nature of the problem. By contrast, our starting point is a Hamiltonian which beautifully leads to an equation of motion valid for all times. Moreover, they treated the acceleration $\ddot{x}(t)$ as the key parameter, ignoring the fact that it is not an observable, in contrast to the applied external force $f(t)$ (which, of course, differs from $M \ddot{x}(t)$ because of the dissipation), which is the basic parameter used in our approach. Since all previous solutions suffered from one problem or another, in section III we present our consistent theory, which led us to a simple second-order differential equation which is not only free of runaway solutions but has no causality problems and is consistent with the optical theorem. Since the basic framework is the same, we also include in this section our treatment of temperature effects on atomic energy and free energy levels.

In section IV, we present the relativistic extension of our theory and in section $\mathrm{V}$ we discuss associated fluctuation and quantum effects. Then, in section VI, we briefly enumerate other applications of our general theory which embraces both radiation reaction and dissipation. Our conclusions are summarized in section VII. 


\section{RADIATION REACTION IN ELECTRODYNAMICS: HISTORICAL SURVEY}

The earliest work on radiation reaction is that of Abraham [1] and Lorentz [2], whose result is summarized in the well-known equation:

$$
M \ddot{x}-M \tau_{e} \dddot{x}=f(t) \quad \text { Abraham-Lorentz, }
$$

where

$$
\tau_{e}=\frac{2 e^{2}}{3 M c^{3}} \simeq 6.25 \times 10^{-24} s .
$$

For a careful discussion of the derivation of this equation, which is "exact," for a point electron, see Jackson [3]. This equation exhibits the well-known problem of runaway solutions: even a small impulsive force acting on an electron at rest results in an exponentially growing displacement. This is made more explicit in the sentence following equation (45). In fact, when $f(t)=0$, equation (18) does not reduce to Newton's equation, as it should. In essence, the problem with the equation might be thought to lie in its derivation which was based on force equations, as distinct from a Hamiltonian. However, as we shall later point out, our exact Hamiltonian approach leads, in the case of a point electron to the same equation (18), making clear that the basic assumption of a point electron is at fault. In fact, both Abraham and Lorentz presented a more systematic discussion by considering both the charge structure of the particle and its self-fields. However, their derivation contained a variety of assumptions and their final result was an infinite expansion, the leading term corresponding to the point electron result given in (18).

Attempts to solve this problem have engaged the efforts of a large number of investigators over the past century. Since, in our view, none of these efforts have been successful, we will just concentrate on presenting the more prominent. First, we note that Born and Infeld [20] attempted to fix the problems associated with the Abraham-Lorentz approach by modifying Maxwell's theory to make it non-linear but, in particular, they encountered problems with quantization.

Dirac [21] attempted to solve the problem by including advanced solutions in addition to retarded solutions but this effort suffered from a violation of causality (so that the acceleration at time $t$ depended on the force acting at times earlier that $t$ ). An attempt by Ivanenko and Sokolov [22] to replace the Abraham-Lorentz equation by an integro-differential equation of motion was also flawed by virtue of introducing a violation of causality. Similar 
remarks apply to the theory of Wheeler and Feynman [23]. See also [24-26]; we discuss the attempts of Eliezer [25] and Landau and Lifshitz [26] in more detail in section III, in connection with our own results.

\section{A CONSISTENT THEORY OF RADIATION REACTION}

The Abraham-Lorentz equation is a result of nineteenth century physics with, moreover, no notion of fluctuations. Almost without exception, discussions of radiation reaction in the past century have relied on the same physics. On the other hand, our contribution has been to recognize that a correct equation is a quantum Langevin equation for which the heat bath is the blackbody radiation field, with fluctuations due to the fluctuations of that field. As we have seen, a consistent derivation of such an equation begins with a Hamiltonian formulation of the dynamics and the laws of thermodynamics impose a powerful constraint on that formulation.

For a nonrelativistic electron (charge $=-e$ ) interacting with the quantum electrodynamic radiation field the Hamiltonian has the form [9]:

$$
H_{\mathrm{QED}}=\frac{1}{2 m}\left(\mathbf{p}+\frac{e}{c} \mathbf{A}\right)^{2}+V(\mathbf{r})+\sum_{\mathbf{k}, s} \hbar \omega_{k} a_{k, s}^{\dagger} a_{k, s}-x f(t)
$$

where the vector potential is given by

$$
\mathbf{A}=\sum_{\mathbf{k}, s} \sqrt{\frac{2 \pi \hbar c^{2}}{\omega_{k} V}} f_{k} \hat{\mathbf{e}}_{\mathbf{k}, s}\left(a_{\mathbf{k}, s}+a_{\mathbf{k}, s}^{\dagger}\right)
$$

Here the symbols have their usual meanings. The quantity $f_{k}$ is the electron form factor (Fourier transform of the electron charge distribution). Without loss of generality, we have taken the form factor as well as the polarization vector $\hat{e}_{k, s}$, to be real. The form factor, which is sometimes called a cutoff factor, must have the property that it is unity up to some large cutoff frequency $\Omega$ beyond which it falls to zero. Then, we showed [9] that, by a unitary transformation that leaves the position operator unchanged, this Hamiltonian can be put into the independent oscillator form (5). Rather than repeating that discussion, we will show how to get the quantum Langevin equation directly from this form of the Hamiltonian. 
The Heisenberg equations of motion are

$$
\begin{aligned}
\dot{\mathbf{r}} & =\frac{1}{i \hbar}\left[\mathbf{r}, H_{\mathrm{QED}}\right]=\frac{\mathbf{p}+\frac{e}{c} \mathbf{A}}{m}, \\
\dot{\mathbf{p}} & =\frac{1}{i \hbar}\left[\mathbf{p}, H_{\mathrm{QED}}\right]=-\frac{\partial V}{\partial \mathbf{r}}+f(t), \\
\dot{a}_{\mathbf{k}, s} & =\frac{1}{i \hbar}\left[a_{\mathbf{k}, s}, H_{\mathrm{QED}}\right]=-i \omega_{k}-i \sqrt{\frac{2 \pi e^{2}}{\hbar \omega_{k} V}} f_{k} \hat{\mathbf{e}}_{\mathbf{k}, s} \cdot \frac{\mathbf{p}+\frac{e}{c} \mathbf{A}}{m} .
\end{aligned}
$$

Eliminating the particle momentum operator between the first two equations, we get the particle equation of motion:

$$
m \ddot{\mathbf{r}}+\frac{\partial V}{\partial \mathbf{r}}=\frac{e}{c} \dot{\mathbf{A}} .
$$

The solution of the last equation can be written

$$
a_{\mathbf{k}, s}(t)=a_{\mathbf{k}, s}^{(h)}(t)-i \sqrt{\frac{2 \pi e^{2}}{\hbar \omega_{k} V}} f_{k} \hat{\mathbf{e}}_{\mathbf{k}, s} \cdot \int_{-\infty}^{t} d t^{\prime} e^{-i \omega_{k}\left(t-t^{\prime}\right)} \dot{\mathbf{r}}\left(t^{\prime}\right)
$$

where $a_{\mathbf{k}, s}^{(h)}(t)$ is the solution of the homogeneous equation, corresponding to free motion of the bath in the absence of the electron. Putting this in the expression (21) for the vector potential, we find

$$
\mathbf{A}(t)=\mathbf{A}^{(h)}(t)-\frac{4 \pi e c}{V} \sum_{\mathbf{k}, s} \frac{f_{k}^{2}}{\omega_{k}} \int_{-\infty}^{t} d t^{\prime} \sin \left[\omega_{k}\left(t-t^{\prime}\right) \hat{\mathbf{e}}_{\mathbf{k}, s} \hat{\mathbf{e}}_{\mathbf{k}, s} \cdot \dot{\mathbf{r}}\left(t^{\prime}\right)\right] .
$$

The sum over $s$ is the sum over the two polarization directions perpendicular to $\mathbf{k}$, so we have $\sum_{s} \hat{\mathbf{e}}_{\mathbf{k}, s} \hat{\mathbf{e}}_{\mathbf{k}, s} \cdot \dot{\mathbf{r}}=\dot{\mathbf{r}}-\hat{\mathbf{k}} \cdot \dot{\mathbf{r}} \hat{\mathbf{k}}$. Next we form the limit of an infinite quantization volume, using the prescription $\sum_{\mathbf{k}} \rightarrow \frac{V}{(2 \pi)^{3}} \int d \mathbf{k}$. With this the particle equation of motion (23) takes the form of a quantum Langevin equation:

$$
m \ddot{\mathbf{r}}+\int_{-\infty}^{t} d t^{\prime} \mu\left(t-t^{\prime}\right) \dot{\mathbf{r}}\left(t^{\prime}\right)+\frac{\partial V}{\partial \mathbf{r}}=\mathbf{F}(t)+f(t),
$$

where the memory function is

$$
\mu(t)=\frac{4 e^{2}}{3 \pi c^{3}} \int_{0}^{\infty} d \omega_{k} \omega_{k}^{2} f_{k}^{2} \cos \left(\omega_{k} t\right) \theta(t),
$$

and the fluctuating operator force is

$$
\mathbf{F}(t)=\frac{e}{c} \dot{\mathbf{A}}^{(h)}(t) .
$$

Note that these expressions for the memory function and the fluctuating force satisfy the general feature that they depend only on the bath parameters, independent of the particle mass and the external potential. 
From the expression (27) for the memory function, we find that the spectral distribution is

$$
\operatorname{Re}\left[\tilde{\mu}\left(\omega+i 0^{+}\right)\right]=\frac{4 \pi e^{2}}{6 \pi c^{3}} \int d \omega_{k} \omega_{k}^{2} f_{k}^{2} \delta\left(\omega-\omega_{k}\right)=\frac{2 e^{2} \omega^{2}}{3 c^{3}} f_{k}^{2} .
$$

The physically significant results for this model should not depend upon details of the electron form factor, subject, of course, to the condition that it be unity up to some large frequency $\Omega$ and falls to zero thereafter. A convenient form which satisfies this condition is

$$
f_{k}^{2}=\frac{\Omega^{2}}{\omega_{k}^{2}+\Omega^{2}}
$$

Using this in the expression (29) for the spectral distribution, the Stieltjes inversion formula (13) gives

$$
\tilde{\mu}(z)=\frac{2 e^{2}}{3 c^{3}} \frac{z \Omega^{2}}{z+i \Omega} .
$$

Finally, with the form (30) for the form factor, the expression (27) for the memory function can be evaluated to give

$$
\mu(t)=\frac{2 e^{2}}{3 c^{3}} \Omega^{2}\left(2 \delta(t)-\Omega e^{-\Omega t}\right) \theta(t) .
$$

Put this in the equation of motion (note $\delta(t) \theta(t)$ is "half" $\delta(t)$ ) with $V(\mathbf{r}, t)=\frac{1}{2} K r^{2}-\mathbf{r} \cdot \mathbf{f}(t)$, corresponding to an external oscillator potential and an applied force $\mathbf{f}(t)$. Then multiply both sides by $e^{\Omega t}$ and differentiate with respect to $t$, to get the equation of motion in the form:

$$
\frac{1}{\Omega} m \dddot{\mathbf{r}}+\left(m+\frac{2 e^{2}}{3 c^{3}} \Omega\right) \ddot{\mathbf{r}}+\frac{1}{\Omega} K \dot{\mathbf{r}}+K \mathbf{r}=\mathbf{f}(t)+\frac{1}{\Omega} \dot{\mathbf{f}}(t)+\mathbf{F}(t)+\frac{1}{\Omega} \dot{\mathbf{F}}(t) .
$$

For motion for which the typical frequencies are much below the cutoff frequency $\Omega$, this becomes of the form for a free particle, but with a renormalized mass:

$$
M=m+\frac{2 e^{2} \Omega}{3 c^{3}}
$$

The mass $M$ is interpreted as the observed mass of the electron, although at ultrahigh frequencies $m$, the bare mass, reappears. Now with this interpretation the bare mass, what we have up till now been calling the electron mass, is no longer observable, but is given in terms of the observed quantities through

$$
m=M\left(1-\Omega \tau_{e}\right),
$$


where $M=9.11 \times 10^{-28} \mathrm{~g}$ and $\tau_{e}=2 e^{2} / 3 M c^{3}=6.25 \times 10^{-24} s$. With this expression for the bare mass, the equation (33) can be written

$$
M\left(\frac{1}{\Omega}-\tau_{e}\right) \dddot{\mathbf{r}}+M \ddot{\mathbf{r}}+\frac{1}{\Omega} K \dot{\mathbf{r}}+K \mathbf{r}=\mathbf{F}(t)+\frac{1}{\Omega} \dot{\mathbf{F}}(t)+\mathbf{f}(t)+\frac{1}{\Omega} \dot{\mathbf{f}}(t) .
$$

Within the framework of our model, this is an exact Heisenberg operator equation of motion.

For the purpose of making contact with the Abraham-Lorentz and other classical equations, in Eq. (36) we will take mean values, set $K=0$ and specialize to one dimension. Thus, the fluctuating force $F(t)$ is eliminated and all quantities are now to be interpreted as classical quantities and we obtain

$$
M\left(\Omega^{-1}-\tau_{e}\right) \dddot{x}(t)+M \ddot{x}(t)=f(t)+\Omega^{-1} \dot{f}(t) .
$$

We immediately see that the Abraham-Lorentz equation (18) follows if we take $\Omega \rightarrow \infty$, corresponding to a point-electron model for the electron. But in this limit the bare mass (35) is negative infinity. This is the source of the runaway solutions that plague that model.

If the bare mass is to be positive the relation (35) puts a constraint on the cutoff frequency:

$$
\Omega \leq \tau_{e}^{-1}
$$

and hence the point electron model, associated with the Abraham-Lorentz equation, is ruled out. The largest possible value of the cutoff consistent with this constraint is $\Omega=\tau_{e}^{-1}$, corresponding to zero bare mass. Choosing this value of the cutoff, the equation of motion (36) becomes

$$
M \ddot{\mathbf{r}}+\tau_{e} K \dot{\mathbf{r}}+K \mathbf{r}=\mathbf{f}(t)+\tau_{e} \dot{\mathbf{f}}(t)+\mathbf{F}(t)+\tau_{e} \dot{\mathbf{F}}(t) .
$$

This is a rather striking exact result in that it is only a second-order equation with the only parameter being $\tau_{e}$. Its form is a result of our choice (30) for the cutoff function with the choice $\Omega=\tau_{e}^{-1}$. Other forms of the cutoff function will give rise to terms on the right hand side of higher order in $\tau_{e}$, but the first order term is the same for all. Since these higher order terms reflect meaningless details of the cutoff function, we feel that the simple equation (39) is the one of choice. It is our key result.

The case usually discussed in the literature corresponds to a free classical particle $(K=0)$ with neglect of fluctuations $(F(t)=0)$ and motion in one dimension, where our equation (39) specializes to the form

$$
M \ddot{x}=f(t)+\tau_{e} \dot{f}(t) . \quad \text { Ford-O'Connell. }
$$


We emphasize that $f(t)$ is a general time-dependent external field. In the particular case where this field is an electric field, we found that $[27,28]$

$$
f(t)=\left(\frac{1}{1+\omega^{2} \tau_{e}^{2}}\right)^{1 / 2} e E(t),
$$

which, except for very large frequencies $\omega$ is essentially $e E(t)$. It is of interest to note that Eliezer [25], whose approach was to postulate what he considered possible solutions, also wrote down (40) with $f(t)=e E(t)$ [see Eliezer's equation (9)]. Landau and Lifshitz [26] later obtained by a method of successive approximations to the Abraham-Lorentz equation a result similar to that of Eliezer. Of course, neither of these investigators realized that all departures from the AL equation require the existence of a charge with structure.

Equation (40) is to be compared with the Abraham-Lorentz equation (18). Indeed, if in that equation we assume that in first approximation the term $-M \tau_{e} \dddot{x}$ is small and can be neglected, we get $M \ddot{x} \cong f(t)$ and $-M \tau_{e} \dddot{x} \cong-\tau_{e} \dot{f}(t)$, giving our equation (40). This is essentially the argument used by Eliezer [25] and by Landau and Lifshitz [26] to get a corresponding result. But, due to the existence of runaway solutions, the term $-M \tau_{e} \dddot{x}$ is never small for all times, so their argument is flawed. On the other hand, as we have seen, our equation (40) is the result of a consistent theory with consistent approximations.

Forming the Fourier transform of equation (39), we can write the solution as

$$
\tilde{\mathbf{r}}(\omega)=\alpha(\omega)[\tilde{\mathbf{f}}(\omega)+\tilde{\mathbf{F}}(\omega)]
$$

where the polarizability is given by

$$
\alpha(\omega)=\frac{1-i \omega \tau_{e}}{-M \omega^{2}+\left(1-i \omega \tau_{e}\right) K} .
$$

As a simple application we can calculate the autocorrelation (17) in the classical limit $(\hbar \rightarrow 0)$. With the polarizability $(43)$ we find

$$
\begin{aligned}
\frac{1}{2}\langle x(t) x(0)+x(0) x(t)\rangle & =\frac{2 k T \tau_{e}}{M \pi} \int_{0}^{\infty} d \omega \frac{\omega^{2}}{\left(\omega_{0}^{2}-\omega^{2}\right)^{2}+\gamma^{2} \omega^{2}} \cos (\omega t) \\
& =\frac{k T}{K} e^{-\gamma|t| / 2}\left[\cos \left(\omega_{1} t\right)-\frac{\gamma}{2 \omega_{1}} \sin \left(\omega_{1}|t|\right)\right]
\end{aligned}
$$

where

$$
\omega_{0}^{2}=\frac{K}{M}, \quad \gamma=\frac{K \tau_{e}}{M}, \quad \omega_{1}=\sqrt{\omega_{0}^{2}-\frac{\gamma^{2}}{4}}
$$


If we make the same calculation with the Abraham-Lorentz equation we find an autocorrelation that grows exponentially in time, a clearly unphysical result which shows that in the presence of fluctuations the problem of runaway solutions is inescapable.

It is well known that the Abraham-Lorentz equation is compatible with the Larmor formula for the radiated power. However, for our equation (40) we find that the total electromagnetic energy radiated from a confined current distribution $[28,29]$ is

$$
W_{\mathrm{R}}=\frac{2 e^{2}}{3 c^{3}} \int_{-\infty}^{\infty} d t\left(\frac{\mathbf{f}(t)}{M}\right)^{2} .
$$

This is our generalization of the Larmor formula. If we make the replacement $\mathbf{f}(t) \rightarrow$ $M \ddot{\mathbf{r}}$, which corresponds to setting $\tau_{e}=\Omega^{-1} \rightarrow 0$, we get the familiar form of the Larmor formula. Thus we see that our expression (46) for the radiated energy is compatible with our form (40) of the equation of motion, just as the Larmor expression is compatible with the Abraham-Lorentz equation. Finally, we should emphasize that for a force $\mathbf{f}(t)$ that is slowly varying on a time scale $\tau_{e}$ the difference between the two expressions is negligibly small but our expression is in terms of the aplied force, as distinct from the theoretically derived acceleration.

At first, one might be surprised that (39) predicts that for a free particle $(K=0)$ there is no radiation if the external force is constant whereas (46) seems to state otherwise. The answer is that (46) was derived, as is usual, with the assumption that $f(t)$ is switched on in the distant past and off in the distant future. For a force that is switched on, is constant for a long time, and then switched off, there is no radiation during the intermediate times of constant force [29], yet the total radiated energy is correctly given by the formula (46). This was verified in an explicit example [30]. With some exceptions, it appears that this simple explanation was often missed in the endless debate surrounding this problem in the past (where, in dealing with the Abraham-Lorentz equation, the argument centred on constant acceleration). The most notable exception was Feynman [31] who states that "- - we have inherited a prejudice that an accelerating charge should radiate - the power radiated by an accelerating charge [the Larmor formula] has led us astray," and he then goes on to discuss the limited validity of the Larmor formula and the fact that "- - it does not suffice to tell us " when," the energy is radiated".

Finally, we point out that our equation (39) is not only free of runaway solutions but is also consistent with the optical theorem and the standard formulas for the Rayleigh and 
Thomson scattering cross sections [27]. Moreover, the corresponding polarizability (43), since it is analytic in the upper half $\omega$ plane, is consistent with the basic physical requirement of causality.

It is also interesting to note that in our first paper in this general area [8], we considered the motion of a charged particle in a radiation field, with the purpose of finding the effect of temperature on atomic energy levels (and, of course, this was the framework we later used for the treatment of radiation from the electron). This followed much earlier work by Knight [32] at a time when it was considered that the effects were so small as to be unobservable. However, using high-precision laser spectroscopic techniques, in a remarkable paper, Hollberg and Hall [33] were able to measure fractional shifts of $\sim 2 \times 10^{-12}$. In our approach, since temperature was involved, we were obliged to consider thermodynamics and, in particular, free energy. As a result, we obtained a striking exact result for the free energy of a quantum oscillator interacting with a blackbody radiation field, which we used to obtain agreement [34] with the experimental results obtained for the energy shifts due to temperature [33]. Later, when a flurry of paper appeared claiming the laws of thermodynamics were invalid in the quantum arena, we were able to show, using this same free energy result, that these claims were in fact incorrect [35].

\section{RADIATION REACTION: RELATIVISTIC THEORY}

Analogous to Dirac's extension of the Abraham-Lorentz equation [21], we have proposed an extension of our equation (39) to the relativistic domain [30]. The form is

$$
M \frac{d u^{\mu}}{d \tau}=\frac{e}{c} F_{\kappa}^{\mu} u^{\kappa}+\tau_{e} \frac{e}{c}\left(\frac{d}{d \tau} F_{\lambda}^{\mu} u^{\lambda}-\frac{1}{c^{2}} u^{\mu} u^{\kappa} \frac{d}{d \tau} F_{\kappa \lambda} u^{\lambda}\right),
$$

where $F^{\mu \nu}$ is the external electromagnetic field tensor and

$$
u^{\mu}=\frac{d x^{\mu}}{d \tau}, \quad d \tau=\frac{1}{c} \sqrt{g_{\mu \nu} d x^{\mu} d x^{\nu}}=\sqrt{1-\frac{v^{2}}{c^{2}}} d t .
$$

This equation is consistent with the constraint

$$
g_{\mu \nu} u^{\mu} u^{\nu}=c^{2}
$$

and in the nonrelativistic limit $(c \rightarrow \infty)$ reduces to our equation (39) for the special case of a free particle $(K=0)$ and for the mean motion with no fluctuation $(F(t)=0)$. Also in 
that limit, the applied force is $\mathbf{f}(t)=e \mathbf{E}(t)$, which tells us that the equation is really only valid for spatially uniform (although possibly time dependent) fields.

It is also of interest to note that our equation of motion (47) can be written in the three-vector form

$$
M \frac{d \gamma \mathbf{v}}{d t}=\mathbf{F}+\tau_{e}\left[\gamma \frac{d \mathbf{F}}{d t}-\frac{\gamma^{3}}{c^{2}}\left(\frac{d \mathbf{v}}{d t} \times(\mathbf{v} \times \mathbf{F})\right)\right] .
$$

Here $\mathbf{v}=\dot{\mathbf{r}}, \gamma=\sqrt{1-v^{2} / c^{2}}$ and

$$
\mathbf{F}=e(\mathbf{E}+\mathbf{v} \times \mathbf{B}),
$$

is the Lorentz force with $\mathbf{E}$ and $\mathbf{B}$ the electric and magnetic fields. We note that the corrections to the non-relativistic results are of order $(v / c)^{2}$, as one might expect.

In [30] we presented an exact solution of this equation for the case of an electron travelling between the plates of a parallel-plate capacitor, for which the electric field is uniform between the plates and zero otherwise. The result shows explicitly the radiation occurs only as the electron enters and leaves the field.

\section{FLUCTUATION AND QUANTUM EFFECTS}

Earlier discussions of radiation reaction were generally based on classical electrodynamics and implicitly assumed that fluctuations could be neglected. For the most part, our discussion above was equivalent with such a classical description, with the fluctuation force eliminated by taking mean values. As we have seen in our example (44), in the classical limit this corresponds to zero temperature, which is not a serious limitation for the classical theory. However, in the quantum theory zero point fluctuations are always present. As an example of the importance of quantum fluctuations, we consider the commutator of the position and velocity. Forming the commutator of $\mathbf{r}$ with the first of equations (22), using the canonical commutation we find when specialized to one dimension, $[x(t), \dot{x}(t)]=i \hbar / m$, where $m$ is the bare mass. However, using the expression (43) for the polarizability, the formula (17) for the non-equal time commutator can be readily evaluated and it is obvious that only the renormalized mass appears. In particular, in the limit as $t^{\prime}$ approaches $t$ from

above or below we find that $\left[x(t), \dot{x}\left(t \pm 0^{+}\right)\right]=i \frac{\hbar}{M}\left(1-\gamma \tau_{e}\right)$, with $M$ the renormalized mass. For a detailed discussion see [36]. 
As another example of a quantum fluctuation phenomenon, consider the mean square displacement of a harmonically bound electron at zero temperature, obtained by setting $t^{\prime}=t$ in the correlation (17) and forming the zero temperature limit. With the expression (43) for $\alpha(\omega)$, we obtain an expression in terms of an integral that is logarithmically divergent. However, inclusion of retardation would make the integral finite with an upper limit approximately equal to $M c^{2} / \hbar$.[37] With this mean square displacement is found to be

$$
\left\langle x^{2}\right\rangle_{T=0} \cong \frac{\hbar}{2 M \omega_{0}}\left(1+\frac{2 \omega_{0} \tau_{e}}{\pi} \log \frac{M c^{2}}{\hbar \omega_{0}}\right) .
$$

Since $\omega_{0} \tau_{e}$ is presumed very small this corresponds to a small increase over the leading factor, which is the mean square width of the oscillator ground state.

Finally, we remark on quantum tunneling in a dissipative system. For most systems of interest it was found that dissipation decreases tunneling rates. However, in the case of the blackbody radiation field, we found that tunneling actually increased [38]. The reason for this exception to the general rule is the presence of mass renormalization.

\section{MISCELLANEOUS APPLICATIONS}

The case of constant friction is of special interest. There the spectral distribution is independent of $\omega: \operatorname{Re}\left[\tilde{\mu}\left(\omega+i 0^{+}\right)\right]=\zeta$, the friction constant. This is frequently referred to as an Ohmic heat bath. The equation (6) then takes the form:

$$
m \ddot{x}+\zeta \dot{x}+V^{\prime}(x)=F(t) .
$$

This is the same form as the original, classical form of the Langevin equation but here, of course, $x$ and $F$ are operators. In this case, since the past motion does not appear, one says there is no memory. On the other hand, the autocorrelation of the quantum mechanical random force $[9,39]$ becomes

$$
\begin{aligned}
\frac{1}{2}\left\langle F(t) F\left(t^{\prime}\right)+F\left(t^{\prime}\right) F(t)\right\rangle & =\frac{\zeta}{\pi} \int_{0}^{\infty} d \omega \hbar \omega \operatorname{coth} \frac{\hbar \omega}{2 k T} \cos \left[\omega\left(t-t^{\prime}\right)\right] \\
& =k T \zeta \frac{d}{d t} \operatorname{coth}\left[\Omega_{\mathrm{T}}\left(t-t^{\prime}\right)\right] \\
& =k T \zeta\left\{-\frac{\Omega_{\mathrm{T}}}{\sinh ^{2}\left[\Omega_{\mathrm{T}}\left(t-t^{\prime}\right)\right]}+2 \delta\left(t-t^{\prime}\right)\right\} .
\end{aligned}
$$


where $\Omega_{\mathrm{T}}=\pi k T / \hbar$. In the limit $\hbar \rightarrow 0$ this becomes the familiar form of classical Brownian motion:

$$
\left\langle F(t) F\left(t^{\prime}\right)\right\rangle \underset{\hbar \rightarrow 0}{\rightarrow} 2 k T \zeta \delta\left(t-t^{\prime}\right), \quad \text { classical. }
$$

The notion of a Markovian system combines two aspects: a stochastic equation with no memory, as in (53), and a delta-function correlation, as in (55). The quantum system is never Markovian, in particular the correlation at zero temperature has no delta-function

The original classical Brownian motion problem corresponds to a free particle $(K=0)$, spectral distribution independent of frequency $\omega$ and $k T>>\hbar \omega$ (absence of quantum effects), in which case the position auto-correlation function is readily calculated, leading to an exact expression for the mean-square displacement, which for large $t$ reduces to the Einstein result (4) for the diffusion constant. However, at low temperatures, non-Markovian quantum effects become important and cannot be neglected. Anomalous diffusion in quantum Brownian motion has also attracted much interest as an explanation of various experiments [40].

Another application of interest relates to Josephson junctions. At first glance one might be puzzled as to how the quantum Langevin equation applies in this case. Actually, although we have used the language of particle motion in our formulation of this description, it should be clear that the description is more general than the language. Thus, the operator $x$ in the quantum Langevin equation (6) can be a generalized displacement operator. By this we mean an operator $x$ such that a term $V(x, t)=-x f(t)$, with $c$-number $f(t)$, added to the microscopic Hamiltonian of the system, results in an added term $f(t)$ on the right-hand side of the equation of motion. One can therefore apply this description to an equation which is formally similar to the Langevin equation but in which the physical meaning of $x$ is different. One must, however, be cautious to check the above generalized displacement property. It turns out that, for Josephson junctions, the phase difference $\phi$ of the superconducting wave function across the junction is such a generalized displacement coordinate. This enabled us to obtain an expression for the power spectrum of the phase fluctuations (generalizing an earlier weak coupling limit result of Josephson) as well the mean square deviation of the phase and the power spectrum of the voltage fluctuations [41].

Turning to the study of small tunnel junctions, here too the quantum Langevin equation proved to be an effective tool in determining the mean-square charge fluctuation on the junction by treating the charge fluctuation as a generalized coordinate [42]. The quan- 
tum Langevin equation has also been used to study quantum transport for a many-body system [43], the advantage being that the separation of frictional (dissipative) and random (fluctuating) forces gives a natural separation between the conductivity and the noise.

In general, the separation of fluctuations (noise) and dissipation in the quantum Langevin equation (6), together with the fluctuation-dissipation theorems, enables us to systematically analyze noise in many different systems. In particular, using (17), we were able to calculate the power spectrum of the coordinate fluctuations in a universal model which we presented for the detection of noise in gravitational wave detector systems [44].

In addition, we found that quantum effects required modification of the famous Onsager (classical) regression hypothesis [18], which states that the regression of fluctuations is governed by the macroscopic equations describing the approach to equilibrium. In other words, the so-called quantum regression theorem is only correct if one makes various approximations [45]. In fact, we claim that the correct generalization of the Onsager regression hypothesis is the fluctuation-dissipation theorem of Callen and Welton [18].

It is also of interest to note that the quantum Langevin equation has been used in a very straightforward manner to analyze the so-called Unruh radiation problem. Its use in this context was first considered by Sciama and co-workers [46] but they used various approximations which left their work open to criticism. However, we carried out an exact analysis of an oscillator (the detector) moving under a constant force with respect to zerotemperature vacuum and coupled to a one-dimensional scalar field [47]. We showed that, contrary to the conclusions reached by Unruh, this system does not radiate despite the fact that it thermalizes at the Unruh temperature.

More recently, the quantum Langevin equation has been used in the general area of mesoscopic systems and quantum information. Its use enabled us to incorporate "entanglement at all times", in contrast to the often used but more approximate master equations. In particular, it enabled us to analyze exactly the decoherence of a Schrödinger cat superposition of Gaussian states, one striking result being the conclusion that decoherence can occur even in the absence of dissipation [48], a result which does not emerge from master equation calculations. It should be noted that one finds many different master equations in the literature. However, even for what is referred to as the "exact master equation" (which are exact only in the sense that they incorporate time-dependent coefficients) [49], we presented what we feel is the most transparant exact solution [50,51]. Our approach started with the Wigner 
function equivalent equation since the Wigner function provides the same information as the corresponding density matrix while making the calculations simpler and more transparent. One striking result we obtained is that the exact master equation problem is equivalent to the Langevin equation for the initial value problem, which was much easier to solve. We found that serious divergences arose at low temperatures [50] and that, even in the high temperature regime [52], problems also exist, notably the fact that the density matrix is not necessarily positive. In addition, Karrlein and Grabert [49] showed that there is no unique master equation, which is connected with the fact that the Onsager regression hypothesis fails in the quantum regime. By contrast, our "entanglement at all times" approach, based on the use of the quantum Langevin equation, has none of these problems [53].

Since entanglement is the essence of various schemes to build small devices for quantum information applications (cryptography, quantum computing, etc.), we used the quantum Langevin equation to examine how disentanglement occurs in two-body systems. In particular, we found that if the temperature is larger than a critical value that disentanglement can occur in the absence of dissipation [54]. However, the effect of temperature is very different for disentanglement than for decoherence because the effect is constant for all time whereas for decoherence the effect increases with time.

\section{CONCLUSIONS}

We have seen that radiation occurs when a system interacts with a heat bath. It is, in essence, a dissipative term (which is not invariant under $t \rightarrow-t$ ) which occurs in the equation of motion of the system. More generally, it occurs in conjunction with fluctuations and the relation between these quantities is expressed in the famous quantum fluctuation-dissipation theorem. In the absence of a heat bath, we still have quantum fluctuations. However, even in the classical case, we have temperatures fluctuations. It is only in the classical case where temperature is neglected do we have zero fluctuations so that the dissipative (radiation reaction) term only appears. This is mainly the arena for discussing radiation

effects in electrodynamics. More generally, as we saw above, there are many systems for which fluctuations play a key role in addition to radiation reaction.

We should remark that we have chosen to write the autocorrelation functions in their symmetric form since we found that this choice led to the most elegant presentation. However, 
clearly other choices are possible, corresponding to the normal and anti-normal ordering of the $a^{+}$(creation) and $a$ (annihilation) operators, but it is clear that in all cases the physical implications are the same, a point emphasized by Milonni in his detailed discussion of QED effects [55].

In our discussion of radiation effects in electrodynamics, we have used as our starting point the universally accepted Hamiltonian of quantum electrodynamics. Treating the blackbody radiation field as a heat bath, this enabled us to obtain an exact equation of motion of an electron in the form of a quantum Langevin equation, in which both dissipation and fluctuations appear. Imposing the requirement that the second law of thermodynamics must be satisfied leads to an equation of motion that is second order in time with no runaway solutions. Thus our equation, in contrast with the well-known Abraham-Lorentz equation, is consistent with physical principles. In particular, causality is preserved at all times and the optical theorem is satisfied.

\section{ACKNOWLEDGMENT}

The author is pleased to acknowledge a longtime collaboration with G. W. Ford (one of the pioneers of this area; see, in particular, [5]) which led to the essential results presented here. This work was partially supported by the National Science Foundation under Grant No. ECCS-1125675.

R. F. O'Connell received a B.Sc. degree (1953) from the National University of Ireland (after which he worked for 4 years as a Telecommunications Engineer), a Ph.D. from the University of Notre Dame (1962), and a D.Sc. (1975) from the National University of Ireland. Before joining Louisiana State University (LSU) in 1964, he worked at the Dublin Institute for Advanced Studies and at IBM. He is presently Boyd Professor (the highest rank in the LSU System) and Professor of Physics at LSU. O'Connell was a NAS-NRC Senior Research Associate at the NASA Institute for Space Studies, New York (1996-1998). He was a visiting scientist at Oak Ridge National Laboratory (1965); Cambridge University (1970); Montana State U. (1971); Lawrence Livermore Laboratory (1973); U. of Trondheim (1975); U. of Paris XI (1975); ICTP Trieste (1976); Laboratoire Aimé Cotton France (1983); Universi-

dade Federal De Santa Catarina, Florianopolis, Brazil (1987) and the Technical University 
of Denmark (1994). He was also an SRC Senior Visiting Fellow at the Universities of Oxford and London in 1975-1976. He spent numerous sojourns as a visiting scientist at the Dublin Institute of Advanced Studies, the Max-Planck Institute for Quantum Optics, Munich and the University of Ulm, Germany. With Barker, obtained the first correct general relativistic calculation of spin and orbital precessions in two-body systems, which was verified by Breton et al. Science 321, 104 (2008). With Jesse Greenstein and Henry, deduced that the Greenwich white dwarf star has a magnetic field which is 300 million times larger than the earth's magnetic field, simultaneously explaining the origin of the famous Minkowski bands. With Wigner, obtained a new position operator. In recent years, with G. W. Ford, demonstrated the broad generality of the use of generalized quantum Langevin techniques for treating dissipative and fluctuation phenomena in quantum mechanics. He was awarded the Sir J. J. Larmor Prize in physics, National University of Ireland (1953); an NAS-NRC Fellowship at the Institute of Space Studies, New York 1966-68; the Distinguished Research Master award, Louisiana State University, 1975; a Senior Visiting Fellowship, from Science Research Council (England), at Oxford University and Queen Mary College, London, January-June, 1976. He was a Board Member, Physical Review A, January 1995-December 2000 and on the Advisory Panel, Journal of Physics A since 2006. He has been a Fellow of the American Physical Society since 1969.

[1] M. Abraham, Prinzipien der Dynamik des Elektrons, Ann. Phys. 10, (1902), p. 105-179.

[2] H. A. Lorentz, The Theory of Electrons and its Applications to the Phenomena of Light and Radiant Heat, 2nd edition, Leipzig, B. G. Teubner, 1916, section 37, p. 48; note 18, p. 252.

[3] J. D. Jackson, Classical Electrodynamics, 3rd edition, Wiley, 1998.

[4] G. W. Ford and R. F. O'Connell, "Radiation Reaction in Electrodynamics and the Elimination of Runaway Solutions," Phys. Lett. A, 157, 217 (1991).

[5] G. W. Ford, M. Kac, and P. Mazur, "Statistical Mechanics of Assemblies of Coupled Oscillations," J. Math. Phys. 6, 504 (1965); G. W. Ford and M. Kac, "On the Quantum Langevin Equation," J. Stat. Phys. 46, 803 (1987).

[6] A. D. Caldeira and A. J. Leggett, " Quantum Tunneling in a Dissipative System," Ann. Phys. (N.Y.) 149, 374 (1983). 
[7] R. P. Feynman and F. L. Vernon, Jr., " The theory of a general quantum system interacting with a linear dissipative system," Ann. Phys. (N.Y.) 24, 118 (1963).

[8] G. W. Ford, J. T. Lewis, and R. F. O'Connell, "Quantum Oscillator in a Blackbody Radiation Field," Phys. Rev. Lett. 55, 2273 (1985).

[9] G. W. Ford, J. T. Lewis, and R. F. O'Connell, "The Quantum Langevin Equation," Phys. Rev. A 37, 4419 (1988).

[10] R. Brown, Philos. Mag 4, 161 (1828); Ibid. 6, 161 (1829).

[11] A. Einstein, Investigations on the Theory of the Brownian Movement (Dover, New York, 1956).

[12] M. Smoluchowski,"Zur kinetischen Theorie der Brownischen Molekularbewegung und der Suspensionen", Annalen der Physik 21 (1906) 756-780.

[13] M. P. Langevin, "Sur la théorie du mouvement brownien," C. R. Hebd Seances Acad. Sci. 146, 530 (1908) [a translation of this article appears in D. S. Lemons and A. Gythiel, "On the theory of Brownian Motion," Am. J. Phys. 65, 1079 (1997)].

[14] S. Chandrasekhar, "Stochastic Problems in Physics and Astronomy," Rev. Mod. Phys. 15 , 1 (1943).

[15] R. Nyquist, "Thermal Agitation of Electric Charge in Conductors," Phys. Rev. 32 , 110 (1928); J. B. Johnson, "Thermal Agitation of Electricity in Conductors," Phys. Rev. 32, 97 (1928).

[16] H. B. Callen and T. A. Welton, " Irreversibility and Generalized Noise," Phys. Rev. 83, 34 (1951); G. W. Ford, J. T. Lewis, and R. F. O'Connell, "Quantum Oscillator in a Blackbody Radiation Field II. Direct calculation of the Energy using the Fluctuation-Dissipation Theorem," Ann. Phys. (N.Y.) 185, 270 (1988).

[17] X. L. Li, G. W. Ford, and R. F. O'Connell, " Correlation in the Langevin Theory of Brownian Motion," Am. J. Phys 61, 924 (1993).

[18] G. W. Ford and R. F. O'Connell, "There is No Quantum Regression Theorem," Phys. Rev. Lett. 77, 798 (1996).

[19] L. Onsager, "Reciprocal Relations in Irreversible Processes, II," Phys. Rev. 38, 2265 (1931).

[20] M. Born and L. Infeld, "Foundations of the new Field Theory," Proc. Roy. Soc. A 144, 425 (1934).

[21] P. A. M. Dirac, "Classical theory of radiating electrons," Proc. Roy. Soc A 167, 148 (1938).

[22] D. Iwanenko and A. Sokolow, Klassische Feldtheorie, Akademie-Verlag, Berlin (1953), trans- 
lated from the Russian edition (1949).

[23] J. A. Wheeler and R. P. Feynman, "Classical Electrodynamics in Terms of Direct Particle Interaction," Rev. Mod. Phys. 21, 3 (1949), p. 425-433.

[24] For example, see the following reviews: A. D. Yaghijian, Relativistic Dynamics of a Charged Sphere, 2nd edition, Springer, 2006; S. Coleman, in Electromagnetism: Paths to Research, ed. D. M. Teplitz, Plenum Press, New York (1982), pp. 183-210; T. Erber, The Classical Theories of Radiation Reaction, Fortsch. d. Phys, 9, 343 (1961).

[25] C. J. Eliezer, "On the classical theory of particles", Proc. R. Soc. London A 194, 543 (1948).

[26] L. D. Landau and E. M. Lifshitz, The Classical Theory of Fields, 4th ed. (ButterworthHeinmann Oxford, 1987).

[27] F. Intravaia, R. Behunin, P. W. Milonni, G. W. Ford, and R. F. O'Connell, "Consistency of a Causal Theory of Radiative Reaction with the Optical Theorem," Phys. Rev. A 84, 035801 (2011).

[28] G. W. Ford and R. F. O'Connell, " Structure Effects on the Radiation Emitted from an Electron," Phys. Rev. A 44, 6386-6387 (1991).

[29] G. W. Ford and R. F. O'Connell, "Total Power Radiated by an Accelerated Charge," Phys. Lett. A 158, 31-32 (1991).

[30] G. W. Ford and R. F. O'Connell, " Relativistic Form of Radiation Reaction," Phys. Lett. A 174, 182-184 (1993).

[31] R. P. Feynman, "Feynman Lectures on Gravitation," Westview Press, 2003, p. 123-124.

[32] P. L. Knight, "Effect of External Fields on the Lamb Shift," J. Phys. A: Gen. Phys. 5, 417 (1972).

[33] L. Hollberg and J. H. Hall, " Measurement of the Shift of Rydberg Energy Levels Induced by Blackbody Radiation," Phys. Rev. Lett 53, 230 (1984).

[34] G. W. Ford, J. T. Lewis, and R. F. O'Connell, "On the Thermodynamics of Quantum Electrodynamic Frequency Shift," J. Phys. B 20, 899 (1987).

[35] G. W. Ford and R. F. O'Connell, "Entropy of a Quantum Oscillator coupled to a Heat Bath and implications for Quantum Thermodynamics," Physica E 29, 82 (2005); ibid. "A Quantum Violation of the Second Law," Phys. Rev. Lett. 96, 020402 (2006).

[36] G. W. Ford and R. F. O'Connell, "Canonical Commutator and Mass Renormalization," J. Stat. Phys. 57, 803 (1989). 
[37] G. W. Ford and W. von Waldenfels." Radiative energy shifts for a nonrelativistic atom," American Journal of Physics 66 (1998) 975-981.

[38] G. W. Ford, J. T. Lewis, and R. F. O'Connell, "Quantum Tunneling in a Blackbody Radiation Field," Phys. Lett. 158, 367 (1991).

[39] G. W. Ford and R. F. O'Connell, " Derivative of the Hyperbolic Cotangent," Nature 380, 113 (1996).

[40] G. W. Ford and R. F. O'Connell, "Anomalous diffusion in quantum Brownian motion with colored noise," Phys. Rev. A 73, 032103 (2006).

[41] G. W. Ford, J. T. Lewis, and R. F. O'Connell, "Dissipative Quantum Tunneling: Quantum Langevin Equation Approach," Phys. Lett. A 128, 29 (1988).

[42] G. Y. Hu and R. F. O'Connell, "Charge Fluctuations and Zero-Bias Resistance in Small Capacitance Tunnel Junctions," Phys. Rev. B 46, 14219 (1992); G. Y. Hu and R. F. O'Connell, "Langevin Equation Analysis of a Small Capacitance Double Junction," Phys. Rev. B 49, 16505 (1994).

[43] G. Y. Hu and R. F. O'Connell, "Quantum Transport for a Many Body System using a Quantum Langevin Equation Approach," Phys. Rev. B 36, 5798 (1987).

[44] R. F. O'Connell, "Noise in Gravitational Wave Detector Suspension Systems: A Universal Model," Phys. Rev. D 64, 022003 (2001).

[45] G. W. Ford and R. F. O'Connell, "Comment on 'The Lax-Onsager Regression "Theorem"",Optics Comm 179, 477 (2000) and in "Ode to a Quantum Physicist," (Elsevier, Amsterdam, 2000).

[46] D. J. Raine, D. W. Sciama, P. Grove, "Does a uniformly accelerated quantum oscillator radiate?," Proc. R. Soc. London A 435, 205 (1991).

[47] G. W. Ford and R. F. O'Connell, "Is there Unruh Radiation?," Phys. Lett. A 350, 17 (2006).

[48] G. W. Ford and R. F. O'Connell, "Decoherence without Dissipation," Phys. Lett. A 286, 87 (2001).

[49] B. L. Hu, Juan Pablo Paz, and Yuhong Zhang, "Quantum Brownian motion in a general environment: Exact master equation with nonlocal dissipation and colored noise," Phys. Rev. D 45, 2843 (1992); R. Karrlein and H. Grabert, "Exact time evolution and master equations for the damped harmonic oscillator," Phys. Rev. E 55, 153 (1997).

[50] G. W. Ford and R. F. O'Connell, "Exact solution of the Hu-Paz-Zhang master equation," 
Phys. Rev. D 64, 105020 (2001).

[51] G. W. Ford and R. F. O'Connell, "Measured quantum probability distribution functions for Brownian motion," Phys. Rev. A 76, 042122 (2007).

[52] G. W. Ford and R. F. O'Connell, "Limitations on the utility of exact master equations".

[53] G. W. Ford, J. T. Lewis, and R. F. O'Connell, "Quantum Measurement and Decoherence," Phys. Rev. A 64, 032101 (2001).

[54] G. W. Ford and R. F. O'Connell, "Disentanglement and decoherence without dissipation at non-zero temperature," Physica Scripta 32, 038112 (2010).

[55] P. W. Milonni, "The Quantum Vacuum: An Introduction to Quantum Electrodynamics," Academic Press, 1993. 